\title{
Knochenmetastasen, Teil 1
}

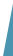
$m$ Laufe seines Berufslebens hat ein Orthopäde durchschnittlich wahrscheinlich nur zwei- bis dreimal die "Chance" sich unmittelbar mit der Diagnostik oder der Therapie eines malignen primären Knochentumors eines von ihm betreuten Patienten zu befassen. Völlig anders ist die Situation bei Knochenmetastasen: Bei einer gegenwärtigen Rate von über $22 \%$ krebsbedingter Todesfälle (Statistisches Bundesamt) und einer Neigung der häufigsten Tumorentitäten (Mamma-Carcinom, Lungen-Carcinom), bevorzugt in das Skelett zu metastasieren, beträgt die Wahrscheinlichkeit, daß ein Orthopäde mit derartigen Fällen im Laufe seines Berufslebens konfrontiert wird, ohne Zweifel $100 \%$. Die unübersehbare Fülle von neuen Erkenntnissen zur Tumorentstehung, Metastasierungsmechanismen, Diagnostik und Therapieverfahren machen eine neue Standortbestimmung sinnvoll. Wo früher mittels bildgebender Verfahren ein Tumorvolumen von weniger als $1 \mathrm{~cm}^{3}$ nicht mehr zuverlässig darstellbar war, und somit der Diagnostik entging, existieren jetzt hochauflösende Schnittbildverfahren, die um ein Vielfaches kleinere Tumorvolumina zuverlässig darstellen können. Mit molekularbiologischen Methoden ist darüber hinaus heute sogar der Nachweis einzelner Tumorzellen im Blut möglich. Viel weniger stürmisch verläuft die Entwicklung auf dem Gebiet der Therapie: Eine Heilung einer Tumorerkrankung im Stadium der Skelettmetastasierung gilt bis auf einige wenige kasuistische Ausnahmen nach wie vor als unmöglich. Demgegenüber wurden zahlreiche neue palliative Therapieverfahren entwickelt, die die Prognose für die betroffenen $\mathrm{Pa}$ tienten in erheblicher Weise günstig beeinflussen können. So tragen neue Verfahren der Chemotherapie, der Strahlentherapie und ergänzend medikamentöse Therapiekonzepte entscheidend zur Steigerung der Le- bensqualität der Patienten bei. Die beiden folgenden Hefte des "Orthopäden“ sollen den Leser auf der Basis einer aktuellen Standortbestimmung unterstützen bei der Entscheidungsfindung, welche diagnostischen und therapeutischen Verfahren situations- und patientengerecht zu wählen sind.

Im ersten Heft stehen Fragen der Metastasenbiologie, der Diagnostik und der nichtinvasiven Therapie im Vordergrund. Vor dem Hintergrund einer $z u$ nehmenden Zahl von Arzthaftungsverfahren erscheint auch für Orthopäden die genaue Kenntnis aller Möglichkeiten der verfügbaren diagnostischen, bildgebenden Verfahren und die Erfolgsraten der nichtoperativen Metastasentherapie unverzichtbar.

Die Vorgehensweise in der Diagnostik ist weitgehend standardisierbar. Dies gilt nicht für die Indikationsstellung der Therapieverfahren. Diese ist allenfalls systematisierbar. Die Zahl der zu berücksichtigenden Einzelfaktoren ist so groß, daß stets eine Individualentscheidung gefällt werden muß

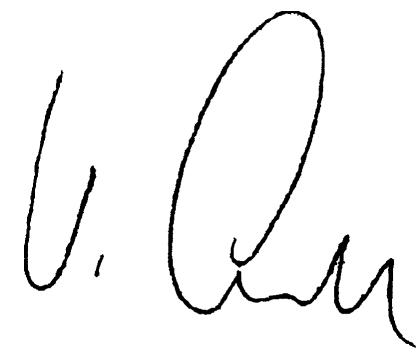

V.Ewerbeck Die Herausgeber 